
\title{
S Research Square
}

\section{TRIM11 promotes the proliferation, migration and chemoresistance of anaplastic thyroid cancer by stabilizing YAP}

Jianing Tang

Wuhan University Zhongnan Hospital

Zelin Tian

Wuhan University Zhongnan Hospital

Xing Liao

wuhan university zhongnan hospital

Qiuxia Cui

Wuhan University Zhongnan Hospital

Gaosong Wu ( $\nabla$ wugaosontj@163.com )

Wuhan University Zhongnan Hospital

\section{Research}

Keywords: anaplastic thyroid cancer, TRIM11, YAP, stabilization, mono-ubiquitination

Posted Date: July 10th, 2020

DOI: https://doi.org/10.21203/rs.3.rs-40875/v1

License: (c) (1) This work is licensed under a Creative Commons Attribution 4.0 International License. Read Full License 


\section{Abstract \\ Background}

Anaplastic thyroid cancer (ATC) is one of the most aggressive and virulent solid tumors. The ubiquitin proteasome system presents in all eukaryotic cells and is essential for cellular homeostasis. While its underlying role in ATC remains largely unclear. TRIM11 is an E3 ubiquitin ligase and has been reported to act as an oncogene in several human cancers. The present study aims to reveal the oncogenic function of TRIM11 in ATC.

\section{Methods}

Western blot was used to measure the protein expression of TRIM11 and YAP, while the YAP target genes were measured by real-time PCR. CCK8 assay was used to detect cell viability; wound-healing assay and transwell assay were used to measure the migration ability of ATC. The xeno-graft tumor model was used for in vivo study. RNA sequencing was analyzed by Ingenuity Pathway Analysis. Protein stability assay was used to detect YAP protein degradation. Immuno-precipitation assay was used to detect the interaction domain between YAP and TRIM11. The ubiquitin-based Immuno-precipitation assays were used to detect the specific ubiquitination manner happened on YAP.

\section{Results}

TRIM11 depletion significantly decreases cell proliferation and migration capabilities of ATC cells, and elevates cell sensitivity to chemotherapy. The effects induced by TRIM11 depletion could be rescued by further YAP overexpression. Depletion TRIM11 decreases YAP protein level and YAP/TEAD target genes, such as CTGF, ANKRD1 and CYR61 in ATC. Immuno-precipitation assay shows that TRIM11 associates with YAP, promoting YAP stabilization possibly via inducing YAP mono-ubiquitination. Further mechanistic analysis indicates that the RING domain of TRIM11 interacts with the WW domain of YAP and promotes its mono-ubiquitination, thus prolongs YAP protein half.

\section{Conclusions}

Our study describes the oncogenic function of TRIM11 in ATC, which acts as a post-translational modulating factor of Hippo pathway. Targeting TRIM11 could be a promising therapeutic method for ATC treatment.

\section{Background}

Thyroid cancer ranks in ninth place in malignancy worldwide. While it is the most commonly diagnosed endocrine malignancy and accounts for more than $90 \%$ of all endocrine cancer cases[1, 2]. Based on the 
histopathological features and the degree of differentiation, thyroid cancer has been divided into three major categories: well-differentiated thyroid cancer (WDTC) which includes papillary thyroid cancer and follicular thyroid cancer; poorly-differentiated thyroid cancer (PDTC) and undifferentiated/anaplastic thyroid cancer (ATC)[3]. Well differentiated thyroid cancer is the most common thyroid cancer, accounting for approximately $90 \%$ of all cases. It is one of the most indolent tumors, which exhibits excellent prognosis with $>98 \% 5$-year survival[4-6]. Anaplastic thyroid cancer is a small subset of thyroid cancer. It accounts for only $2-5 \%$ of thyroid cancer[7-9]. Although ATC is rare, it is responsible for $40-50 \%$ of total thyroid cancer-related deaths due to its high proliferation rate and invasive behavior. Median survival of which is 3 to 5 months. 1-year survival rate is estimated at 10-20\%[10-12]. ATCs rarely respond to the conventional treatment such as radioactive iodine and chemotherapy. In recent years, understanding of this disease has been improved, however, due to the aggressive nature of ATC, treatment options remain limited and largely ineffective [13].

Ubiquitination is an important posttranslational modification, which is a central component of the cellular protein-degradation machinery and essential for cellular homeostasis[14]. The ubiquitin proteasome pathway (UPP) present in all eukaryotic cells. It is an extensive and complex protein degradation pathway and regulates various biological processes such as cell survival, apoptosis, DNA repair, cell-cycle progression, signal transduction, antigen presentation and protein turnover by the proteasome[15-17]. Ubiquitination involves the sequential transfer of an ubiquitin molecule mediated by three enzymes: ubiquitin-activating enzyme (E1), an ubiquitin conjugating enzyme (E2) and an ubiquitin ligase (E3)[18]. E3s are involved in many cellular processes and various types of cancer. They act as oncogenes or tumor suppressors according to the nature of their substrates via regulating protein stability and subcellular localization[19]. However, the underlying role of E3 ligase in thyroid cancer, especially in ATC remains largely unclear.

Substrates may be ubiquitinated as mono-ubiquitination, multiubiquitination (mono-ubiquitination of several residues), or polyubiquitination. Polyubiquitination chain can be linked at seven lysine positions $(6,11,27,29,33,48$, and 63$)$ of ubiquitin[20]. These modifications may result in a different cellular outcome. Mono-ubiquitination can regulate processes such as DNA repair, gene expression, endocytosis, and protein stability [21-23]. Multi-ubiquitination is associated with receptor endocytosis[23]. K11- or K48-linked polyubiquitination generally leads to proteasomal degradation[24], whereas K63-linked ubiquitin chains can function in signaling and endocytosis[21]. The role of K6-, K27-, K29-, and K33-linked ubiquitin chains are less clear. Polyubiquitination and mono-ubiquitination compete to modify the substrate protein to regulate its stability, and mono-ubiquitination is more likely to confer the substrate stability by inhibiting its polyubiquitination and degradation.

TRIM11 is an E3 ubiquitin ligase and belongs to the TRIM family. The oncogenic function of TRIM11 has been reported in a variety of human cancers, including glioma, lung cancer and hepatocellular carcinoma cancer[25-28]. In the present study, we noticed that TRIM11 was associated with more aggressive phenotype of ATC. In the further analysis, we found TRIM11 was possibly to enhance YAP stability, which could be a promising therapeutic target for anaplastic thyroid cancer treatment. 


\section{Materials And Methods}

\section{Cell culture}

HEK293T cells and the anaplastic thyroid cancer cell lines CAL62, and KHM-5M were obtained from the American Type Culture Collection (ATCC; Rockville, MD, USA). All cell lines are possese of cell line authentication via Short Tandem Repeat (STR), which is performed through PowerPlex 21 system, turning out that The STR data keep consistent with its in ATCC. HEK293T and CAL62 were maintained in Dulbecco's Modified Eagle's Medium (DMEM, 41965, Life Technologies) supplemented with $10 \%$ fetal bovine serum (FBS). KHM-5M cells were cultured in RPMI/1640 medium (42401, Life Technologies) supplemented with $10 \% \mathrm{FBS}$. All cells were cultured at $37^{\circ} \mathrm{C}$ in a humidified $5 \% \mathrm{CO}_{2}$ incubator.

\section{Plasmids and RNA inference}

The wild type TRIM11, YAP and their mutant constructs were obtained from Hanbio Biotechnology Co. Ltd. (Shanghai, China). The HA-K6, -K11, -K27, -K29, -K33, -K48, -K63, -K0 and -Ub plasmids were acquired from Addgene. Plasmids were transfected using Lipofectamin 2000 (1662298, Invitrogen). Small interfering RNAs were used for specific gene knocking-down. The TRIM11 siRNA sequences used were: siRNA \#1: 5'-CCAACCGCCCGCUUGCUAA-3'; siRNA \#2: 5'- GGGUGAGUUCGAGCGUCUU-3'. And YAP siRNA sequences were: siRNA \#1: 5'- GCUCAUUCCUCUCCAGCUU-3'; siRNA \#2: 5'- CACCUAUCACUCUCGAGAU-3'. SiRNAs were transfected using RNAiMAX reagent (13778150, invitrogen).

\section{RNA extraction and qPCR analysis}

The total RNA was extracted from the cancer cells using the RNeasy plus mini kits (Qiagen, Germany). Reverse transcription was performed using the PrimeScript RT Master Mix (Takara, Japan). qRT-PCR was performed using the SYBR green mix (Toyobo, Japan) with the CFX96TM Real-time PCR Detection System (Bio-Rad, USA). The $2^{-\triangle \Delta C t}$ method was used to calculate the relative expression. 36B4 was used for internal control. All assays were performed in triplicates.

\section{Proliferation, cell cycle and colony formation assay}

CAL62 and KHM-5M were treated with SiTRIM11 or SiControl in 6-well plates. Forty-eight hours after transfection, the cells were counted and 2000 cells were seeded in 96-well plates. Cell viability was measured using Cell Counting Kit-8 (CCK8) every 24 hours. Cells transfected with SiTRIM11 or SiControl for 48 hours were stained with propidium iodide (Multisciences, China) and analyzed by flow cytometer (Beckman, USA), The cell cycle phases were determined by relative DNA content. For the clone formation 
assay, cells were treated with $50 \mathrm{nM}$ TRIM11 siRNA or $50 \mathrm{nM}$ siControl, after 48 hours, the cells were typsinized and seeded (1-1.5×103 cells/well) in 6-well plates and maintained in complete medium for 2 weeks. The cells were fixed with $4 \%$ paraformaldehyde for 2 hours, and stained with $1 \%$ crystal violet. EdU incorporation assay was performed as our previously described.

\section{Wound-healing assay}

Cells were seeded into 6-well plates and transfected with TRIM11 siRNA or siControl. When full confluent, the cell layer was scratched with a $200 \mu \mathrm{l}$ sterile pipette tip and washed with PBS. Cells were maintained in the medium containing 1\% FBS and wound distance was measured every 24 hours.

\section{Transwell migration assay}

Migration capability was measured using 24-well transwell chamber systems (Corning, USA) with $8.0 \mu \mathrm{m}$ pore size. Cells were seeded in upper chamber insert and cultured in serum-free medium. The bottom chambers were filled with $10 \%$ fetal bovine serum medium. After incubated at $37^{\circ} \mathrm{C}$ for 24 hours, the cells on the lower surface of the membrane were fixed with $4 \%$ paraformaldehyde and stained with crystal violet. The membranes were placed under an inverted phase contrast microscope and imaged to count the migrated cells. Three independent experiments were conducted.

\section{Luciferase assay}

The Dual-Luciferase Reporter kit (Promega, Germany) was used to measure the luciferase activity of YAPluciferase reporter. The YAP-luciferase reporter and Renilla plasmid were transfected together the into the cells. Luciferase activity was detected after $24 \mathrm{~h}$.

\section{Immunofluorescence assay}

CAL62 and KHM-5M cells cultured on $14 \mathrm{~mm}$ slides in 24-well plates were fixed in $4 \%$ paraformaldehyde at room temperature for 30 minutes. After washing with PBS for 3 times, the cells were blocked with $10 \%$ goat serum and incubated with primary antibodies against YAP (mouse, Santa Cruz), and TRIM11 (rat, Proteintech) at $4{ }^{\circ} \mathrm{C}$ overnight. Followed by incubating with FITC- and Cy3-conjugated secondary antibodies. The images were examined with EVOS ${ }^{\mathrm{TM}}$ M5000 Imaging System. 
BALB/c nude mice aged 3 weeks were obtained from Beijing HFK Bioscience Co., Ltd. in Beijing, China. $1 \times 10^{6}$ CAL62 cells were injected to each mouse. The mice were maintained in a temperature and humidity-controlled and specific pathogen-free environment in the laboratory animal facility of Zhongnan Hospital of Wuhan University. Tumor sizes were measured every 5 days until the end of the experiment. The experiments were performed under the protocols approved by ethnic committee of Zhongnan Hospital of Wuhan University.

\section{Co-immunoprecipitation assay}

Total cell lysis of CAL62 were precleared with rabbit IgG for $2 \mathrm{~h}$ and the immunoprecipitated with YAP (Proteintech,13584-1-AP) or TRIM11 (Proteintech, 10851-1-AP) antibody overnight as previously described[29], while rabbit IgG (Santa Cruz) was used as the negative control. The bounded protein was analyzed by Anti-YAP or Anti-TRIM11 antibody.

\section{Protein stability assays}

CAL62 and KHM-5M cells were seeded in 24-well plates and transfected with siTRIM11 or siControl. After $24 \mathrm{~h}$, cells were treated with $100 \mu \mathrm{M}$ cycloheximide (MCE) for indicated time points. Western blot was performed to detect YAP degradation.

\section{Western blot analysis}

The anaplastic thyroid cancer cells were lysed with RIPA extraction reagent (Beyotime, China) supplemented with protease inhibitors (Sigma-Aldrich, USA). Total protein was separated using 10-12.5\% sodium dodecyl sulfate polyacrylamide gel electrophoresis and transferred to $0.45 \mu \mathrm{m}$ PVDF membrane (Millipore, USA). Primary antibodies were YAP (Proteintech, 13584-1-AP), TRIM11 (Proteintech, 10851-1AP), HA (Proteintech, 51064-2-AP), Myc (Proteintech, 60003-2-Ig), Flag (Proteintech, 20543-1-AP), GAPDH (Proteintech, 60004-1-lg) antibodies. Bands were visualized using an enhanced chemiluminescence (ECL) kit (Boster, China) and detected by ChemiDoc XRS+ Imaging System (Bio-Rad).

\section{RNA sequence analysis}

The RNA sequence analysis (siControl and siTRIM11) was performed by Beijing Genomic Institute (BGI). The RNA sequence data are deposited in the SRA database, which a are available at www.ncbi.nlm.nih.gov/bioproject/PRJNA609252/ 


\section{Statistical analysis}

Student's t test and one-way ANOVA were used to compare 2 and more groups respectively. Multiple comparison with Bonferroni correction was performed when appropriate. A P value $<0.05$ was considered as statistically significant and all tests were two-tailed. All statistical tests were performed with Prism 7.0 (GraphPad, USA).

\section{Results}

\section{TRIM11 depletion inhibits anaplastic thyroid cancer cell proliferation and migration}

We first tested TRIM11 expression level in four kinds of cell lines, including two kinds of papillary thyroid cancer cell lines (B-cpap and TPC-1), two kinds of follicular thyroid cancer cell lines (FTC113 and FTC238), three kinds of anaplastic thyroid cancer cell lines (CAL62, KHM-5M, and 8505C) and a normal thyroid epithelial cell line (Nthy-ori3-1). Our results indicated that mRNA level of TRIM11 was relatively higher in anaplastic thyroid cancer cell lines (Figure S1). Then we chose two anaplastic thyroid cell lines, CAL62 and KHM-5M to investigate the potential functions of TRIM11 in anaplastic thyroid cancer.

TRIM11 silence significantly decreased cell proliferation and inhibited G1-S phase transition in both the two cell lines (Figure 1A, B). Knockdown of TRIM11 also decreased the clone formation of CAL62 and KHM-5M cells (Figure 1C). Consistently, siRNA-mediated TRIM11 depletion significantly inhibited DNA synthesis as evaluated by Edu incorporation assay (Figure 1D, E). Wound-healing and transwell assays indicated that depletion of TRIM11 dramatically decreased the migration of CAL62 and KHM-5M cells (Figure 1F-H).

\section{TRIM11 depletion inhibits Hippo signaling pathway activity}

We next silenced TRIM11 expression in CAL62 cells and examined transcriptional profiles by RNA-seq to further approach the function of TRIM11 in anaplastic thyroid cancer. Compared with the siControl group, YAP target genes (CTGF, CYR61 and ANKRD1) were significantly decreased in SiTRIM11 group, and we noticed that differentially expressed genes were mainly enriched in Hippo signaling pathway (Figure 2A, B). Since YAP is the key effector of Hippo pathway, we then evaluated YAP protein levels in CAL62 and $\mathrm{KHM}-5 \mathrm{M}$ cells using two non-overlapping siRNA targeting TRIM11(Figure $2 \mathrm{C}$ ). Our results showed that TRIM11 depletion significantly decreased YAP protein level as well as its target gene expression (Figure 2D-F). In agreement, YAP-luciferase reporter activity was strongly suppressed by TRIM11 depletion (Figure 2G).

\section{TRIM11 associates with YAP and enhances YAP stability}


Results of immunostaining indicated that YAP and TRIM11 were located in both cytoplasm and nucleus (Figure 3A). Endogenous TRIM11 and YAP proteins were co-immunoprecipitated from lysates of CAL62 cells (Figure 3B). GST-pull-down assay showed that TRIM11 interacted with YAP in vitro (Figure S2). TRIM11 depletion significantly decreased YAP protein level, while in the presence of the proteasome inhibitor MG132, YAP protein level was not further regulated by TRIM11(Figure 3C). To prove that TRIM11 regulates YAP stability, we treated ATC cells using the protein synthesis inhibitor cycloheximide, the half time of YAP was markedly shortened in ATC cells depleted with TRIM11(Figure 3D, E).

\section{Mapping of the binding region between TRIM11 and YAP}

YAP has three functional domains: one TEAD transcription factor-binding domain (TBD), one (YAP-WW1) or two (YAP-WW1 and YAP-WW2) WW domains and one trans-activation domain (TAD) [30]. The deletion mutants of YAP were constructed as follows: $\triangle T B D$ (YAP 171-504), $\triangle T A D$ (YAP 1-292), $\triangle T B D+\triangle W W$ (YAP 292-504), and $\triangle W W+\triangle T A D$ (YAP 1-171) (Figure 4A). As a member of the tripartite motif (TRIM) family, TRIM11 is composed of a RING, a B-box type 1 and a B-box type 2, a coiled-coil region, and a Cterminal PRY-SPRY (PS) motif. We constructed TRIM11 deletion mutants lacking each of the individual domains $(\triangle \mathrm{R}, \triangle \mathrm{BB}, \triangle \mathrm{CC}$ and $\triangle \mathrm{PS})$ to identify the domain(s) of TRIM11 that mediates the interaction with YAP (Figure 4B). Co-IP assay indicated that the RING domain of TRIM11 interacted with YAP WW domain (Figure 4C, D).

\section{TRIM11 stabilizes YAP possibly through mono-ubiquitination}

Ubiquitination assay was performed using a series of ubiquitin mutants. The results indicated TRIM11 significantly increased the mono-ubiquitinated YAP levels while decreased K11- and K48-linked polyubiquitination on YAP protein (Figure S3). In order to identify the functional domain of TRIM11 to modulate YAP ubiquitination, TRIM11 and its deletion mutants were transfected into HEK293T cells together with YAP. TRIM11- $\triangle R$ completely lost its ability of promotion YAP mono-ubiquitination and inhibition of K11- and K48-linked polyubiquitination. While TRIM11- $\triangle \mathrm{BB}, \triangle \mathrm{CC}$ and $-\triangle \mathrm{PS}$ retained this ability (Figure S4).

Since RING domain is essential for TRIM11 function, we change two conserved Cys resides involved in Zn2+ binding (Cys16 and Cys19) in the RING domain to Ala (TRIM11-2CA) (Figure 5A). Ectopic expression of wildtype TRIM11, but not TRIM11-2CA, resulted in YAP elevation in a dose-dependent manner (Figure 5B). The decrease of YAP induced by TRIM11 depletion could be reversed by overexpression of wildtype TRIM11, while TRIM11-2CA had no such effect (Figure 5C). In addition, TRIM11-2CA exhibited a significantly reduced ability to interact with YAP and lost the ability to enhance YAP stability (Figure 5D, E). Ubiquitination assay indicated that TRIM11-2CA lost the ability to promote 
YAP mono-ubiquitination and inhibit K11- and K48-linked YAP polyubiquitination (Figure 5F-I). Collectively, these results demonstrate that TRIM11 regulates YAP via a direct protein-protein interaction that involves the WW domain of YAP and the RING domain of TRIM11.

\section{TRIM11 promotes anaplastic thyroid cancer progression via YAP}

The results identified above suggested that TRIM11 might exert its function through YAP. The oncogenic function of YAP has been validated in most solid tumors. However, its role in anaplastic thyroid cancer has not been revealed. We then depleted YAP in CAL62 and KHM-5M cells. YAP depletion dramatically inhibited cell proliferation and migration (Figure S5). To further verify whether TRIM11 promoted cell proliferation and migration in a YAP-dependent manner, we overexpressed YAP in TRIM11 depletion cells and performed a rescue experiment. YAP overexpression largely recovered the suppressive effects induced by TRIM11 depletion (Figure 6A-E), suggesting that TRIM11 may promote anaplastic thyroid cancer proliferation and migration by regulating YAP. We then used xenograft mice models to further investigate the role of TRIM11 in tumor growth. Our data indicated that depletion of TRIM11 or YAP by lentivirus-based shRNA inhibited tumor growth, while the overexpression of YAP in TRIM11-knockdown cells partly recovered tumor growth (Figure 6F).

\section{TRIM11 regulates response of anaplastic cancer cells to chemotherapy through YAP}

Doxorubicin is commonly used for ATC. The activity of YAP is associated with chemoresistance in various types of malignancies including ovarian cancer, breast cancer, and hepatocellular carcinoma. Since YAP is stabilized by TRIM11, we then examined whether inhibition of TRIM11 affects cell response to doxorubicin. Cells treated with TRIM11 siRNA or YAP siRNA were more sensitive to doxorubicin, and the effects induced by TRIM11 depletion could be abolished by YAP overexpression. To confirm the antichemotherapy function of TRIM11 in vivo, CAL62 cells overexpressing an empty vector, TRIM11 or shRNA targeting YAP were xenografted into nude mice. Tumor growth of the control CAL62 cells was significantly reduced by doxorubicin treatment, while tumor growth of cells overexpressing TRIM11 was much more rapidly than the control cells under the treatment of doxorubicin. Furthermore, depletion of YAP abolished the chemoresistance effect induced by TRIM11 overexpression (Figure S6).

\section{Discussion}

TRIM11 is a member of Tripartite Motif Containing (TRIM) proteins characterized by an N-terminal TRIM/RBCC motif. This motif contains a RING domain, 1 or 2 B-box motifs and a coiled-coil region (RBCC). Besides the N-terminal RBCC domain, TRIM11 contains a C-terminal PRY-SPRY (PS) motif[31]. In lung cancer, TRIM11 is reported to promote cell proliferation and invasion via activating the PI3K/AJT pathway[27]. Consistently, TRIM11 is a component in PHLPP1/AKT signaling pathway in human 
chordoma cells. Expression of TRIM11 is upregulated in chordomas tissues and promotes chordoma cells proliferation[32]. In hepatocellular carcinoma, TRIM11exerts its oncogenic effects through inhibition of P53[33]. In addition, TRIM11 can suppress the anti-tumor effect of proteotoxic stress drugs through cooperating with HSF1[34]. Thus, identifying novel substrate is essential for understanding TRIM11 biology and its implication in tumorigenesis and drug resistance.

The present study indicates YAP activation is important in anaplastic thyroid cancer. YAP depletion dramatically inhibited cell proliferation and migration of ATC. YAP is the evolutionarily conserved key element of the Hippo pathway, which mediate the its function through regulation gene transcription[35]. The Hippo pathway is a newly identified pathway which is highly conserved in mammals. This pathway is known to regulate organ size and tissue growth through a delicate balance between cell proliferation and apoptosis[36]. Accumulating studies indicates the prominent role of the Hippo pathway in tumorigenesis. Elevated activity of this pathway has been observed in various types of cancer: including breast, lung, liver, colon, and others[37, 38]. Hippo pathway may have an important role in the initiation and progression of cancer, YAP activation results in cell transformation and tumor development[39]. Elevated YAP expression promotes cell survival, proliferation, migration, and invasion. Cells can escape contact inhibition upon YAP activation[37]. The activity of YAP is mainly regulated by the MST1/2Lats $1 / 2$ kinase cascade. LATS1/2 directly phosphorylates YAP on multiple sites, resulting in interaction with 14-3-3 protein and cytoplasmic retention[40]. Besides the mechanisms regulating its phosphorylation and localization, YAP can be controlled by other post-translational modification such as ubiquitination. For instance, Fbxw7 regulates YAP protein stability by targeting YAP for ubiquitination and proteasomal degradation in hepatocellular carcinoma[41]; SHARPIN and RNF187 promote YAP degradation via inducing YAP K48-dependent poly-ubiquitination[42, 43]. Deubiquitinating enzymes (DUB) also regulate the stability of YAP protein in human cancers. In breast cancer, USP9X deubiquitinates and stabilizes YAP to promote breast cancer cell proliferation and chemoresistance to therapeutic drugs[44]. USP47 functions as a DUB for YAP in colorectal cancer, USP47 elevation leads to stabilization of YAP and promotes colorectal cancer cell proliferation[45]. DUB3 regulates the protein stability of multiple components of Hippo pathway, including the LATS kinases, the E3 ligase ITCH and the AMOT family proteins, which in turn induces YAP turnover[46]. Thus, Ubiquitination and deubiquitination are important for maintaining the function of Hippo pathway. In this study, we observed that YAP can be modified by both mono-and polyubiquitination, and mono-ubiquitination was more likely to confer the stability of YAP by inhibiting its polyubiquitination and degradation.

Anaplastic thyroid cancer is one of the most aggressive and virulent solid tumors. There exists no effective or standard therapy for the treatment of anaplastic thyroid cancer. Therefore, it is an urgent issue to explore the underlying molecule mechanisms involved in the initiation and progression of anaplastic thyroid cancer. More novel candidate targets are needed to improve the treatment decisions. Here, we found TRIM11 may be a potential therapeutic target of anaplastic thyroid cancer. In the present study, we examined the biological function of TRIM11 using anaplastic thyroid cancer cell lines. TRIM11 depletion significantly inhibited cell proliferation and induced G1 phase arrest. Transwell assay demonstrated that the capability of migration was decreased upon TRIM11 silence. And the suppression 
effects were reversed by overexpressing YAP. Global gene analysis based on RNA-seq indicated that Hippo pathway was significantly suppressed upon TRIM11 depletion. In addition, TRIM11 depletion could significantly decrease YAP protein level as well YAP-luciferase reporter gene activity. As TRIM11 is an E3 ubiquitin ligase, we further analyzed whether TRIM11 could directly bind to YAP and modulate its protein stability. We performed co-ip analysis to identify the association between YAP and TRIM11, the results indicated that RING domain of TRIM11 is essential for the interaction with the WW domain of YAP. Upon inhibition of protein synthesis by cycloheximide, TRIM11 depletion significantly decreased YAP protein half time in CAL62 and KHM-5M cells. To further analysis the underlying mechanisms, a series of mutant ubiquitin were used to identify the linkage of ubiquitin chain. We observed that TRIM11 significantly increased the mono-ubiquitinated YAP while decreased K11- and K48-linked polyubiquitination on YAP protein. Furthermore, TRIM11-2CA lost the ability to promote YAP mono-ubiquitination and inhibit K11and K48-linked YAP polyubiquitination.

\section{Conclusions}

Collectively, TRIM11 regulates YAP via a direct protein-protein interaction that involves the WW domain of YAP and the RING domain of TRIM11 and this stabilization effect may depend on its monoubiquitination modification. Thus, targeting TRIM11 could be a promising strategy or drug target for anaplastic thyroid cancer.

\section{Declarations}

\section{Ethics approval and consent to participate}

The research was carried out according to the World Medical Association Declaration of Helsinki and was approved by the Ethics Committee at Zhongnan Hospital of Wuhan University.

\section{Consent for publication}

Not applicable

\section{Availability of data and materials}

The RNA sequence data are available at www.ncbi.nlm.nih.gov/bioproject/PRJNA609252/

\section{Competing interests}

The authors have no conflicts of interest 


\section{Funding}

This project was funded by the Research and Development Fund of Zhongnan Hospital of Wuhan University (Wu, No.20170101).

\section{Author's contribution}

JT performed most of the bench work. ZT and XL participated in the modification and prognosis analysis of the manuscript. GW supervised the process of the study and wrote the manuscript. All authors read and approved the final manuscript.

\section{Acknowledgements}

We thank Pro. Ting Zhuang and Jian Zhu (Henan Key Laboratory of immunology and targeted therapy, Xinxiang Medical University) for their invaluable help and stimulating discussion during the course of the study.

\section{References}

1. Li Z, Zhang Y, Wang R, Zou K, Zou L. Genetic alterations in anaplastic thyroid carcinoma and targeted therapies. Experimental therapeutic medicine. 2019;18:2369-77.

2. Bray F, Ferlay J, Soerjomataram I, Siegel RL, Torre LA, Jemal A. Global cancer statistics 2018: GLOBOCAN estimates of incidence and mortality worldwide for 36 cancers in 185 countries. CA: a cancer journal for clinicians 2018.

3. O'Neill JP, Shaha AR. Anaplastic thyroid cancer. Oral Oncol. 2013;49:702-6.

4. Lim H, Devesa SS, Sosa JA, Check D, Kitahara CM. Trends in Thyroid Cancer Incidence and Mortality in the United States, 1974-2013. Jama. 2017;317:1338-48.

5. Yu XM, Schneider DF, Leverson G, Chen H, Sippel RS. Follicular variant of papillary thyroid carcinoma is a unique clinical entity: a population-based study of 10,740 cases. Thyroid. 2013;23:1263-8.

6. Saini S, Tulla K, Maker AV, Burman KD, Prabhakar BS. Therapeutic advances in anaplastic thyroid cancer: a current perspective. Mol Cancer. 2018;17:154.

7. Kasaian K, Wiseman SM, Walker BA, Schein JE, Zhao Y, Hirst M, et al. The genomic and transcriptomic landscape of anaplastic thyroid cancer: implications for therapy. BMC Cancer. 2015;15:984.

8. Gilliland FD, Hunt WC, Morris DM, Key CR. Prognostic factors for thyroid carcinoma. A populationbased study of 15,698 cases from the Surveillance, Epidemiology and End Results (SEER) program 
1973-1991. Cancer. 1997;79:564-73.

9. Li M, Milas M, Nasr C, Brainard JA, Khan MJ, Burkey BB, et al. Anaplastic thyroid cancer in young patients: a contemporary review. Am J Otolaryngol. 2013;34:636-40.

10. Nagaiah G, Hossain A, Mooney CJ, Parmentier J, Remick SC. Anaplastic thyroid cancer: a review of epidemiology, pathogenesis, and treatment. Journal of oncology 2011; 2011: 542358.

11. Kebebew E, Greenspan FS, Clark OH, Woeber KA, McMillan A. Anaplastic thyroid carcinoma. Treatment outcome and prognostic factors. Cancer. 2005;103:1330-5.

12. Are C, Shaha AR. Anaplastic thyroid carcinoma: biology, pathogenesis, prognostic factors, and treatment approaches. Ann Surg Oncol. 2006;13:453-64.

13. Sasanakietkul T, Murtha TD, Javid M, Korah R, Carling T. Epigenetic modifications in poorly differentiated and anaplastic thyroid cancer. Molecular cellular endocrinology. 2018;469:23-37.

14. Fu L, Cui CP, Zhang $X$, Zhang $L$. The functions and regulation of Smurfs in cancers. Seminars in cancer biology 2019.

15. Glickman $\mathrm{MH}$, Ciechanover A. The ubiquitin-proteasome proteolytic pathway: destruction for the sake of construction. Physiological reviews. 2002;82:373-428.

16. Schwartz AL, Ciechanover A. Targeting proteins for destruction by the ubiquitin system: implications for human pathobiology. Annu Rev Pharmacol Toxicol. 2009;49:73-96.

17. Manasanch EE, Orlowski RZ. Proteasome inhibitors in cancer therapy. Nature reviews Clinical oncology. 2017;14:417-33.

18. Deshaies RJ, Joazeiro CA. RING domain E3 ubiquitin ligases. Annual review of biochemistry. 2009;78:399-434.

19. Mansour MA. Ubiquitination. Friend and foe in cancer. Int J Biochem Cell Biol. 2018;101:80-93.

20. Scott D, Oldham NJ, Strachan J, Searle MS, Layfield R. Ubiquitin-binding domains: mechanisms of ubiquitin recognition and use as tools to investigate ubiquitin-modified proteomes. Proteomics. 2015;15:844-61.

21. Passmore LA, Barford D. Getting into position: the catalytic mechanisms of protein ubiquitylation. Biochem J. 2004;379:513-25.

22. Haglund K, Di Fiore PP, Dikic I. Distinct monoubiquitin signals in receptor endocytosis. Trends Biochem Sci. 2003;28:598-603.

23. Haglund K, Sigismund S, Polo S, Szymkiewicz I, Di Fiore PP, Dikic I. Multiple monoubiquitination of RTKs is sufficient for their endocytosis and degradation. Nat Cell Biol. 2003;5:461-6.

24. Jin L, Williamson A, Banerjee S, Philipp I, Rape M. Mechanism of ubiquitin-chain formation by the human anaphase-promoting complex. Cell. 2008;133:653-65.

25. Chen Y, Li L, Qian X, Ge Y, Xu G. High expression of TRIM11 correlates with poor prognosis in patients with hepatocellular carcinoma. Clinics research in hepatology gastroenterology. 2017;41:190-6.

26. Di K, Linskey ME, Bota DA. TRIM11 is overexpressed in high-grade gliomas and promotes proliferation, invasion, migration and glial tumor growth. Oncogene. 2013;32:5038-47. 
27. Wang X, Shi W, Shi H, Lu S, Wang K, Sun C, et al. TRIM11 overexpression promotes proliferation, migration and invasion of lung cancer cells. Journal of experimental clinical cancer research: CR. 2016;35:100.

28. Zhang Z, Xu C, Zhang X, Huang L, Zheng C, Chen H, et al. TRIM11 Upregulation Contributes to Proliferation, Invasion, and EMT of Hepatocellular Carcinoma Cells. Oncology research. 2017;25:691-9.

29. Zhang A, Wang W, Chen Z, Pang D, Zhou X, Lu K, et al. SHARPIN Inhibits Esophageal Squamous Cell Carcinoma Progression by Modulating Hippo Signaling. Neoplasia (New York NY). 2019;22:76-85.

30. Pobbati AV, Hong W. Emerging roles of TEAD transcription factors and its coactivators in cancers. Cancer Biol Ther. 2013;14:390-8.

31. Chen L, Zhu G, Johns EM, Yang X. TRIM11 activates the proteasome and promotes overall protein degradation by regulating USP14. Nature communications. 2018;9:1223.

32. Wang B, Wang G, Wang Q, Zhu Z, Wang Y, Chen K, et al. Silencing of TRIM11 suppresses the tumorigenicity of chordoma cells through improving the activity of PHLPP1/AKT. Cancer cell international. 2019;19:284.

33. Liu J, Rao J, Lou X, Zhai J, Ni Z, Wang X. Upregulated TRIM11 Exerts its Oncogenic Effects in Hepatocellular Carcinoma Through Inhibition of P53. Cellular physiology biochemistry: international journal of experimental cellular physiology biochemistry pharmacology. 2017;44:255-66.

34. Chen L, Yang X. TRIM11 cooperates with HSF1 to suppress the anti-tumor effect of proteotoxic stress drugs. Cell cycle (Georgetown Tex). 2019;18:60-8.

35. Yu FX, Zhao B, Guan KL. Hippo Pathway in Organ Size Control, Tissue Homeostasis, and Cancer. Cell. 2015;163:811-28.

36. Mo JS, Park HW, Guan KL. The Hippo signaling pathway in stem cell biology and cancer. EMBO Rep. 2014;15:642-56.

37. Chan SW, Lim CJ, Guo K, Ng CP, Lee I, Hunziker W, et al. A role for TAZ in migration, invasion, and tumorigenesis of breast cancer cells. Cancer research. 2008;68:2592-8.

38. Chen HY, Yu SL, Ho BC, Su KY, Hsu YC, Chang CS, et al. R331W Missense Mutation of Oncogene YAP1 Is a Germline Risk Allele for Lung Adenocarcinoma With Medical Actionability. Journal of clinical oncology: official journal of the American Society of Clinical Oncology. 2015;33:2303-10.

39. Camargo FD, Gokhale S, Johnnidis JB, Fu D, Bell GW, Jaenisch R, et al. YAP1 increases organ size and expands undifferentiated progenitor cells. Current biology: CB. 2007;17:2054-60.

40. Zhou D, Conrad C, Xia F, Park JS, Payer B, Yin Y, et al. Mst1 and Mst2 maintain hepatocyte quiescence and suppress hepatocellular carcinoma development through inactivation of the Yap1 oncogene. Cancer cell. 2009;16:425-38.

41. Tu K, Yang W, Li C, Zheng X, Lu Z, Guo C, et al. Fbxw7 is an independent prognostic marker and induces apoptosis and growth arrest by regulating YAP abundance in hepatocellular carcinoma. Mol Cancer. 2014;13:110. 
42. Zhang A, Wang W, Chen Z, Pang D, Zhou X, Lu K, et al. SHARPIN Inhibits Esophageal Squamous Cell Carcinoma Progression by Modulating Hippo Signaling. Neoplasia (New York NY). 2020;22:76-85.

43. Wang Z, Kong Q, Su P, Duan M, Xue M, Li X, et al. Regulation of Hippo signaling and triple negative breast cancer progression by an ubiquitin ligase RNF187. Oncogenesis. 2020;9:36.

44. Li L, Liu T, Li Y, Wu C, Luo K, Yin Y, et al. The deubiquitinase USP9X promotes tumor cell survival and confers chemoresistance through YAP1 stabilization. Oncogene. 2018;37:2422-31.

45. Pan B, Yang Y, Li J, Wang Y, Fang C, Yu FX, et al. USP47-mediated deubiquitination and stabilization of YAP contributes to the progression of colorectal cancer. Protein cell. 2020;11:138-43.

46. Nguyen HT, Kugler JM, Cohen SM. DUB3 Deubiquitylating Enzymes Regulate Hippo Pathway Activity by Regulating the Stability of ITCH, LATS and AMOT Proteins. PloS one. 2017;12:e0169587.

\section{Supporting Information}

Figure S1. Relative expression level of TRIM11 thyroid cancer cell lines.

Figure S2. Pulldown assay reveals direct interaction between TRIM11 and YAP. CAL62 cells transfected with Flag-TRIM11 were lysed and lysates incubated with GST-YAP or GST protein. The interacted TRIM11 was detected via western blot.

Figure S3. TRIM11 increases the mono-ubiquitinated YAP while decreases K11- and K48-dependent polyubiquitination on YAP protein. (A-I). HEK293 cells were transfected with $2 \mu \mathrm{g}$ Myc-YAP plasmid, 0.5 $\mu \mathrm{g}$ Flag-TRIM11 plasmids, and $0.5 \mu \mathrm{g}$ HA-K6, -K11, -K27, -K29, -K33, -K48, -K63, -K0 or -Ub plasmids. The cell extracts were immunoprecipitated with HA antibody. The specific polyubiquitinated and monoubiquitinated YAP were detected via western blotting analysis.

Figure S4. TRIM11 increases the mono-ubiquitinated YAP and decreases K11- and K48-dependent polyubiquitination on YAP protein via its RING domain. (A-D). HEK293 cells were transfected with $2 \mu \mathrm{g}$

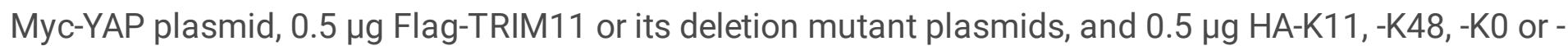
Ub plasmids. The cell extracts were immunoprecipitated with $\mathrm{HA}$ antibody. The specific polyubiquitinated and mono-ubiquitinated YAP were detected via western blotting analysis.

Figure S5. YAP depletion inhibits anaplastic thyroid cancer cell proliferation and migration. (A). YAP depletion inhibits the cell proliferation in anaplastic thyroid cancer cells. (B, C). Representative images of EdU assay of anaplastic thyroid cancer cells. (D). YAP depletion decreases clone formation capability of 
anaplastic thyroid cancer cells. (E). Wound-healing assay of anaplastic thyroid cancer cells. (F). Transwell migration assay of anaplastic thyroid cancer cells.

$\star, P$ value $<0.05 ; * *, P$ value $<0.01 ; * * *, P$ value $<0.001$

Figure S6. TRIM11 regulates the anaplastic cancer cells response to chemotherapy. (A-C). CAL62 Cells expressing indicated constructs were treated with doxorubicin, and cell survival was determined. (D-F). KHM-5M Cells expressing indicated constructs were treated with doxorubicin, and cell survival was determined. (G). In vivo xenografts generated from CAL62 cells expressing an empty vector, TRIM11 or YAP-targeting shRNA and treated with doxorubicin.

\section{Figures}

A

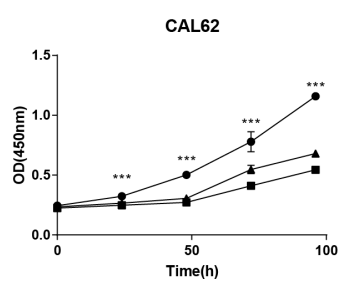

C
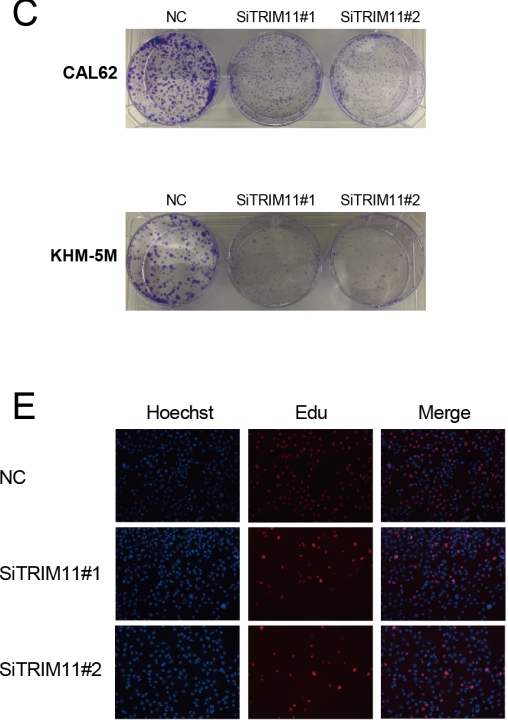

$\mathrm{H}$

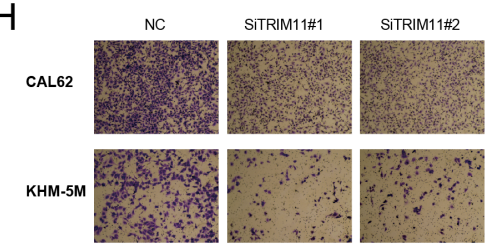

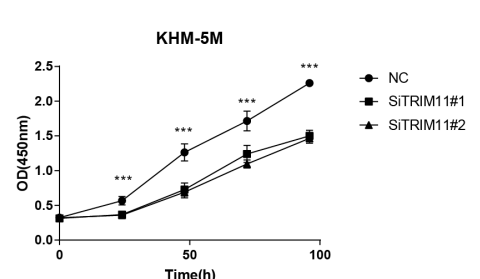

CAL62
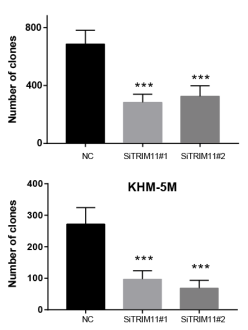

D
$B$
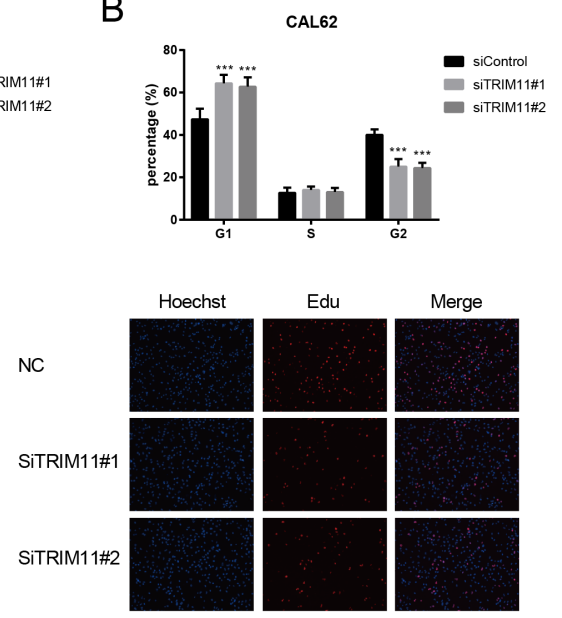

F

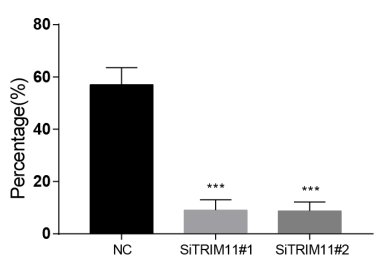

G

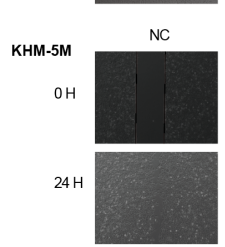

KHM-5M

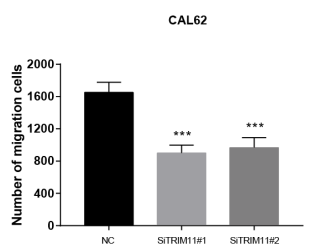

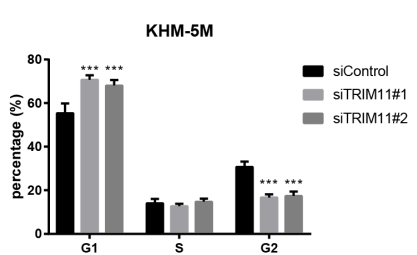

CAL62

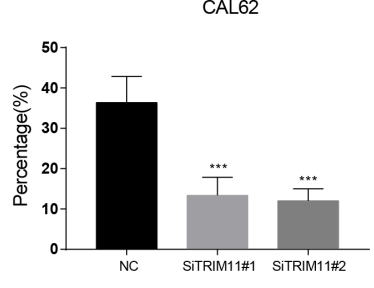

CAL62

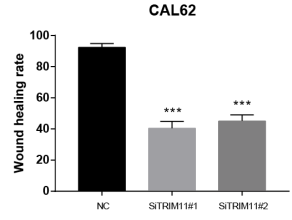

HM-5M

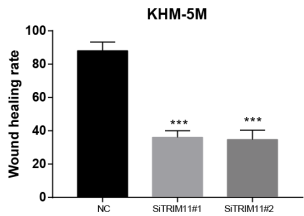

Figure 1 
TRIM11 depletion inhibits anaplastic thyroid cancer cell proliferation and migration. (A). TRIM11 depletion inhibits the cell proliferation in anaplastic thyroid cancer cells. (B). TRIM11 depletion induces G1 cell cycle arrest in anaplastic thyroid cancer cells. (C). TRIM11 depletion decreases clone formation capability of anaplastic thyroid cancer cells. (D, E). Representative images of EdU assay of anaplastic thyroid cancer cells. $(F, G)$. Wound-healing assay of anaplastic thyroid cancer cells. $(H)$. Transwell migration assay of anaplastic thyroid cancer cells. ${ }^{*}, \mathrm{P}$ value $<0.05 ; \star \star$, P value $<0.01$; $* \star \star, ~ P$ value $<$ $0.001 ; * \star \star *, P$ value $<0.0001$
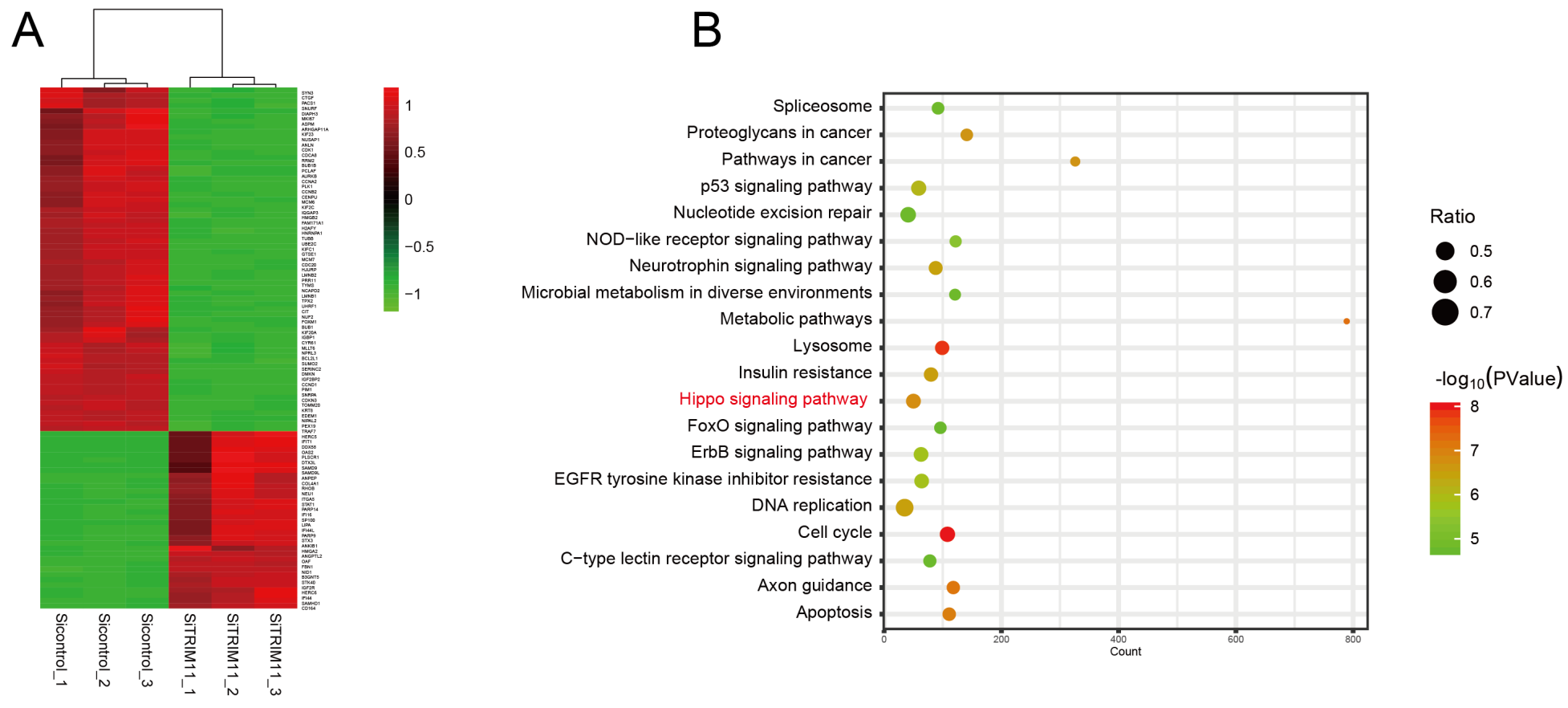

C
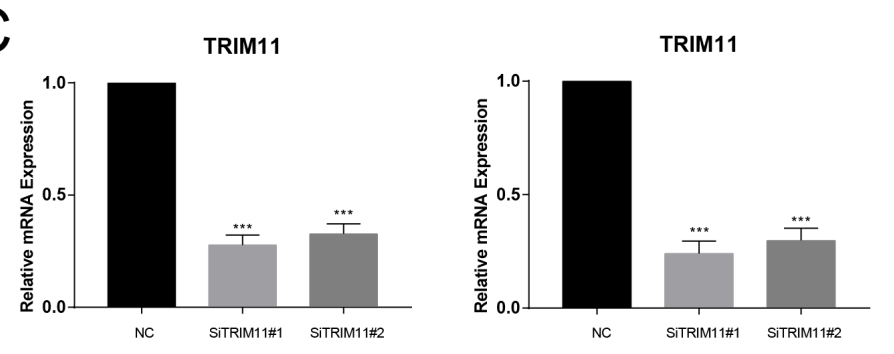

E

CAL62

F

KHM-5M
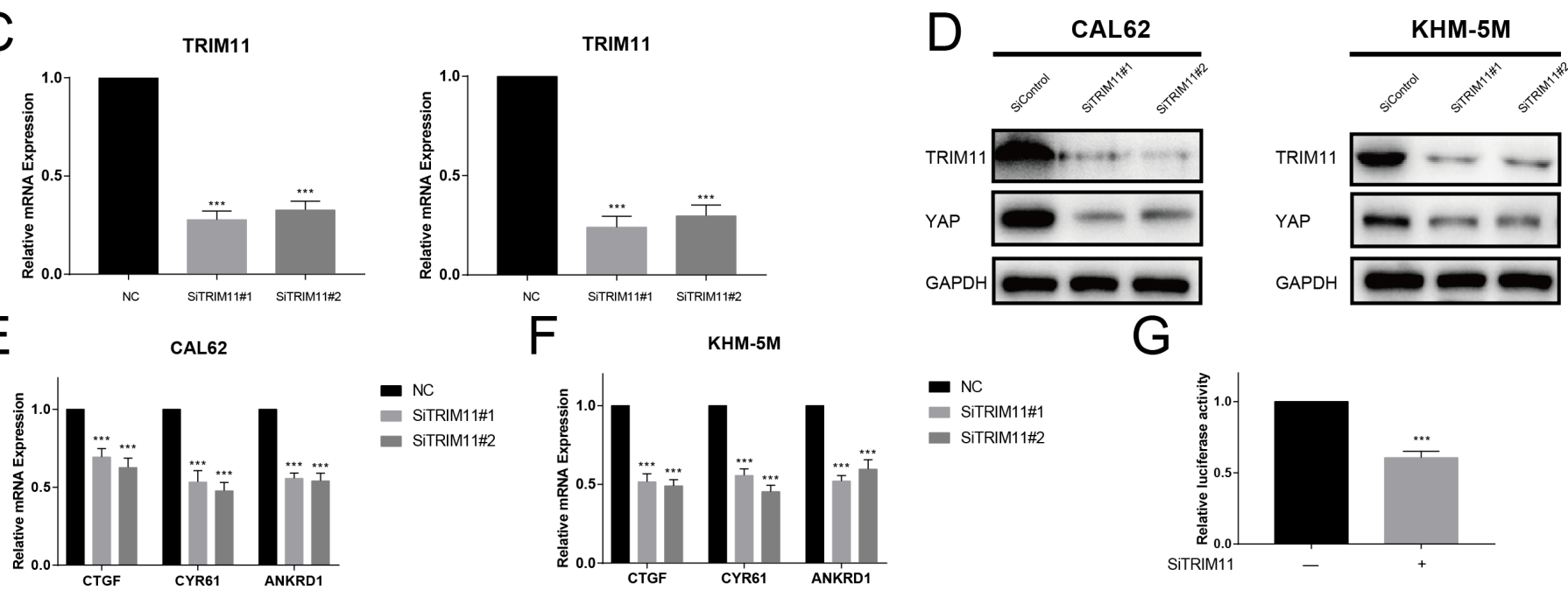

G
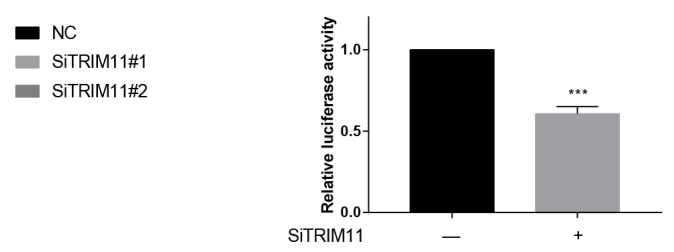

Figure 2

TRIM11 depletion decreases Hippo signaling activity in anaplastic thyroid cancer cells. (A). Heatmap of top 100 differentially expressed genes. (B). Pathway enrichment of differentially expressed genes. (C). TRIM11 depletion effect by two different siRNA oligos. anaplastic thyroid cancer cells are transfected with two independent TRIM11 siRNAs or siControl. After 48 h, TRIM11 mRNA levels are determined by qRT-PCR. 36B4 was used as internal control. (D). TRIM11 depletion decreases YAP protein level. (E, F). 
TRIM11 depletion decreases YAP target genes using two different siRNA oligos. (G). TRIM11 depletion affects YAP-luciferase activity. CAL62 cells were transfected with SiTRIM11 or SiControl together with YAP-luciferase reporter plasmid. Luciferase activity was measured $48 \mathrm{~h}$ after transfection. *, P value < $0.05 ; * \star, P$ value $<0.01 ; * \star *, P$ value $<0.001$
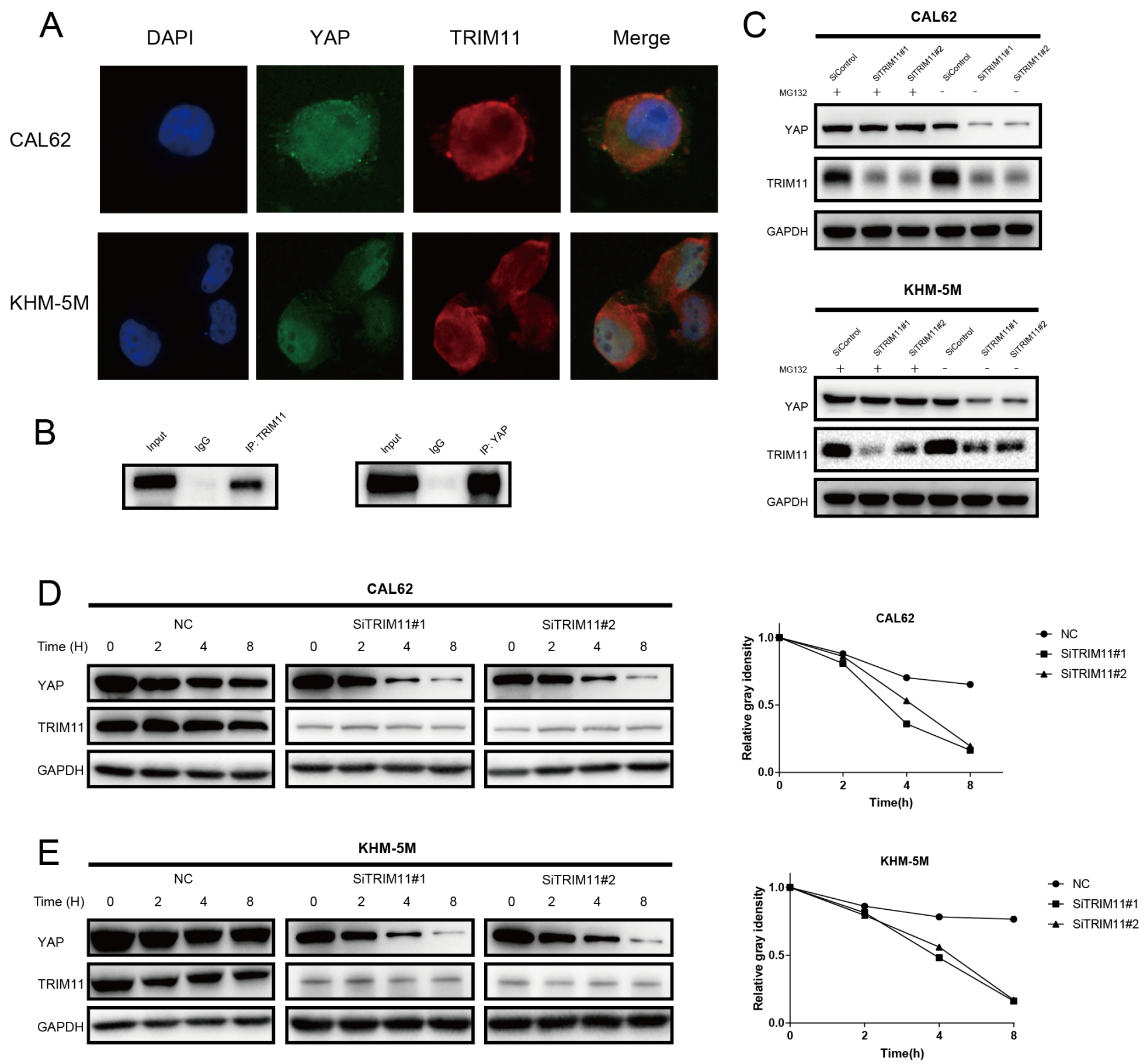

Figure 3

TRIM11 associates with YAP and increases its stability. (A). An immunofluorescence assay demonstrated that TRIM11 and YAP at least partially colocalized in CAL62 and KHM-5M cells. (B). Co-IP assay reveals association between endogenous TRIM11 and YAP in CAL62 cells. CAL62 cells were harvested with RIPA lysis buffer. Co-IP was performed using antibody as indicated. (C). In the presence of the proteasome 
inhibitor MG132, depletion of TRIM11 did not further decrease YAP protein levels. Anaplastic thyroid cancer cells were transfected with siTRIM11 or siControl. After $48 \mathrm{~h}$, cells were treated with $10 \mathrm{nM} \mathrm{MG} 132$ / vehicle for $6 \mathrm{~h}$, cell lysates were prepared for western blot analysis. (D, E). TRIM11 depletion decreases YAP half-life in anaplastic thyroid cancer cells. Anaplastic thyroid cancer cells were transfected with siTRIM11 or siControl. After $48 \mathrm{~h}$, cells were treated with $100 \mu \mathrm{M}$ cycloheximide for indicated times. Cell lysates were prepared for western blot analysis.

A

\begin{tabular}{|c|c|c|c|}
\hline YAP 1-504 & TBD & WW & TA \\
\hline YAP 1-171 & TBD & & \\
\hline YAP 1-292 & TBD & WW & \\
\hline YAP 171-504 & & WW & TA \\
\hline YAP 292-504 & & & TA \\
\hline
\end{tabular}

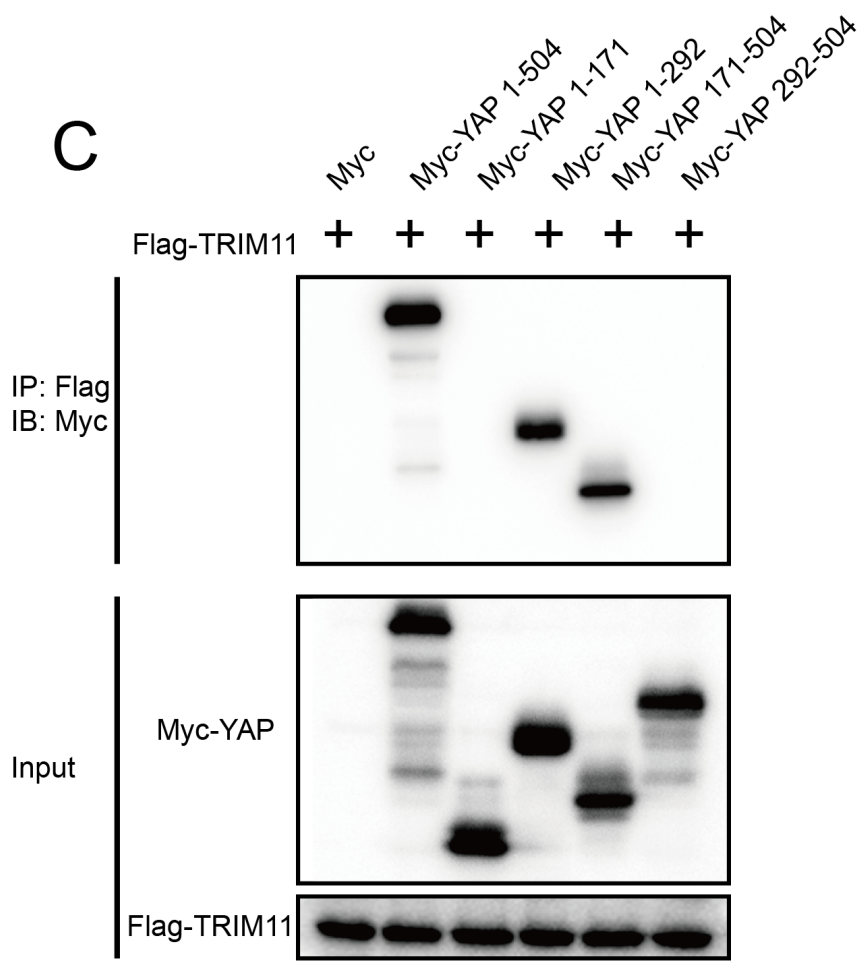

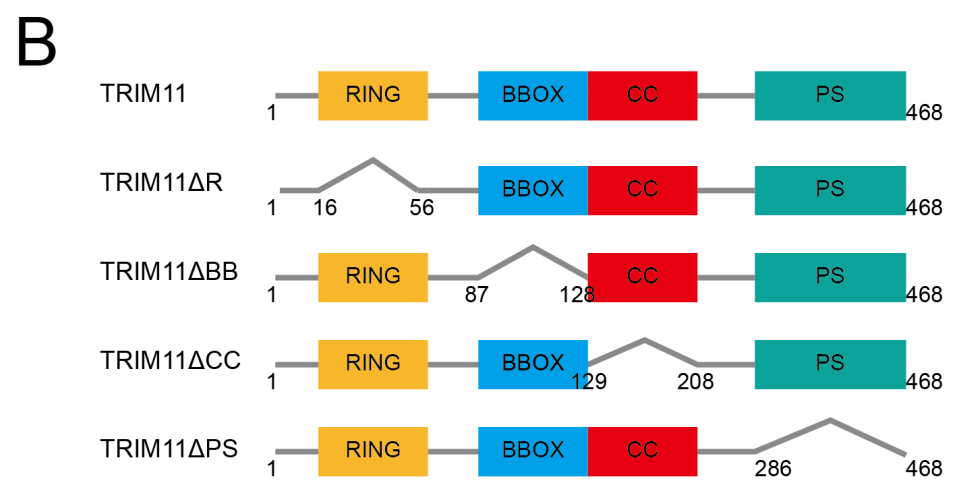

$\mathrm{D}$

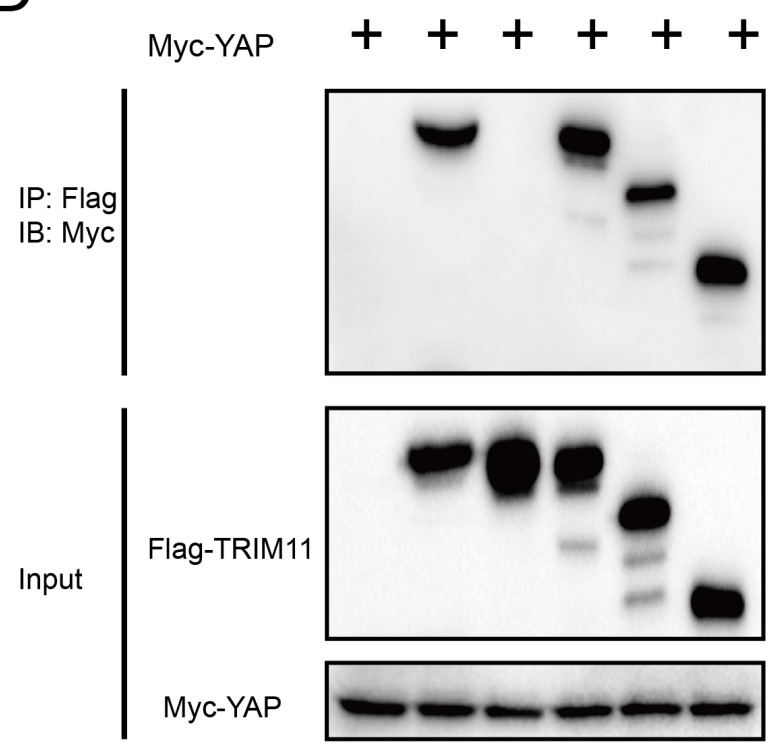

\section{Figure 4}

TRIM11 associates with YAP WW domain through its RING domain. (A, B). YAP and TRIM11 domain structure and deletion mutants used in the study. (C). TRIM11 interacts with YAP through its WW domain. HEK293 cells were transfected with $2 \mu \mathrm{g}$ Flag-TRIM11 together with Myc-YAP alpha full length or mutants. After $24 \mathrm{~h}$, cells were harvested with NP-40 lysis buffer. Co-IP was performed using Flag antibody. The possible interacted YAP domains were detected by Myc antibody. (D). RING domain is required for TRIM11 to interaction with YAP. HEK293 cells were transfected with 2 ug Myc-YAP alpha 
together with Flag-TRIM11 full length or mutants. After 24 h, cells were harvested with NP-40 lysis buffer. Co-IP was performed using Myc antibody. The possible interacted TRIM11 domains were detected by Flag antibody.

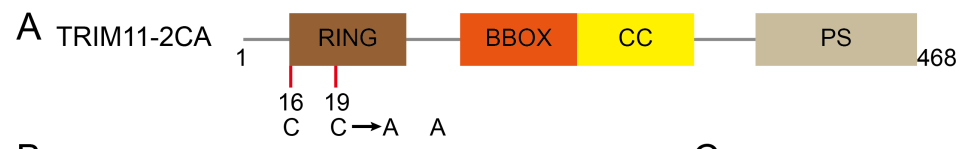

B

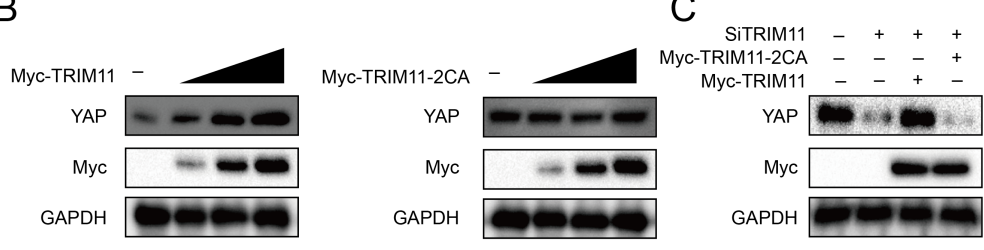

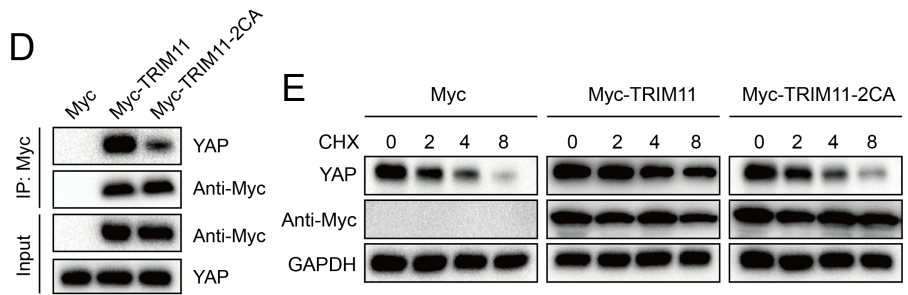
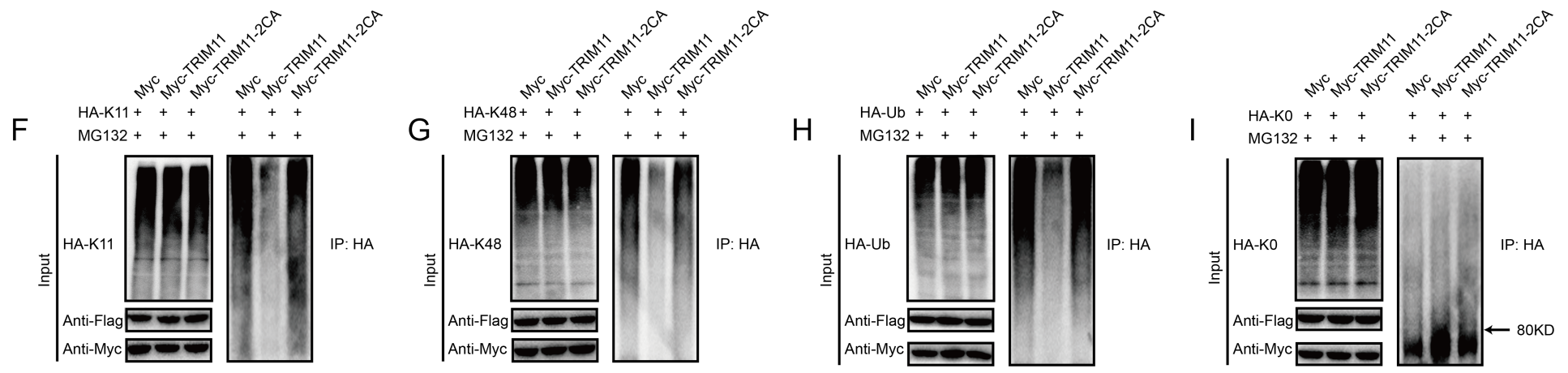

Figure 5

TRIM11 stabilizes YAP though its RING domain. (A). Schematic diagram of TRIM11-2CA. (B). Increasing amounts of TRIM11 WT or 2CA were transfected into CAL62 cells and YAP expression was detected. (C). TRIM11 WT or 2CA was introduced into CAL62 cells together with TRIM11 siRNA. YAP levels were measured. (B). Interaction of Myc-TRIM11-2CA proteins with endogenous YAP in CAL62 cells was analyzed by co-IP assay. (E). HEK293 cells transfected with the indicated plasmids were treated with 100 $\mu \mathrm{M}$ cycloheximide, and collected at the indicated times for western blot. (F-I). TRIM11-2CA does not modulate poly / mono-ubiquitination of YAP protein. 


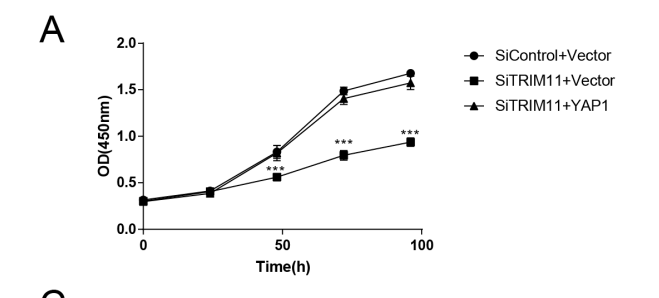

B
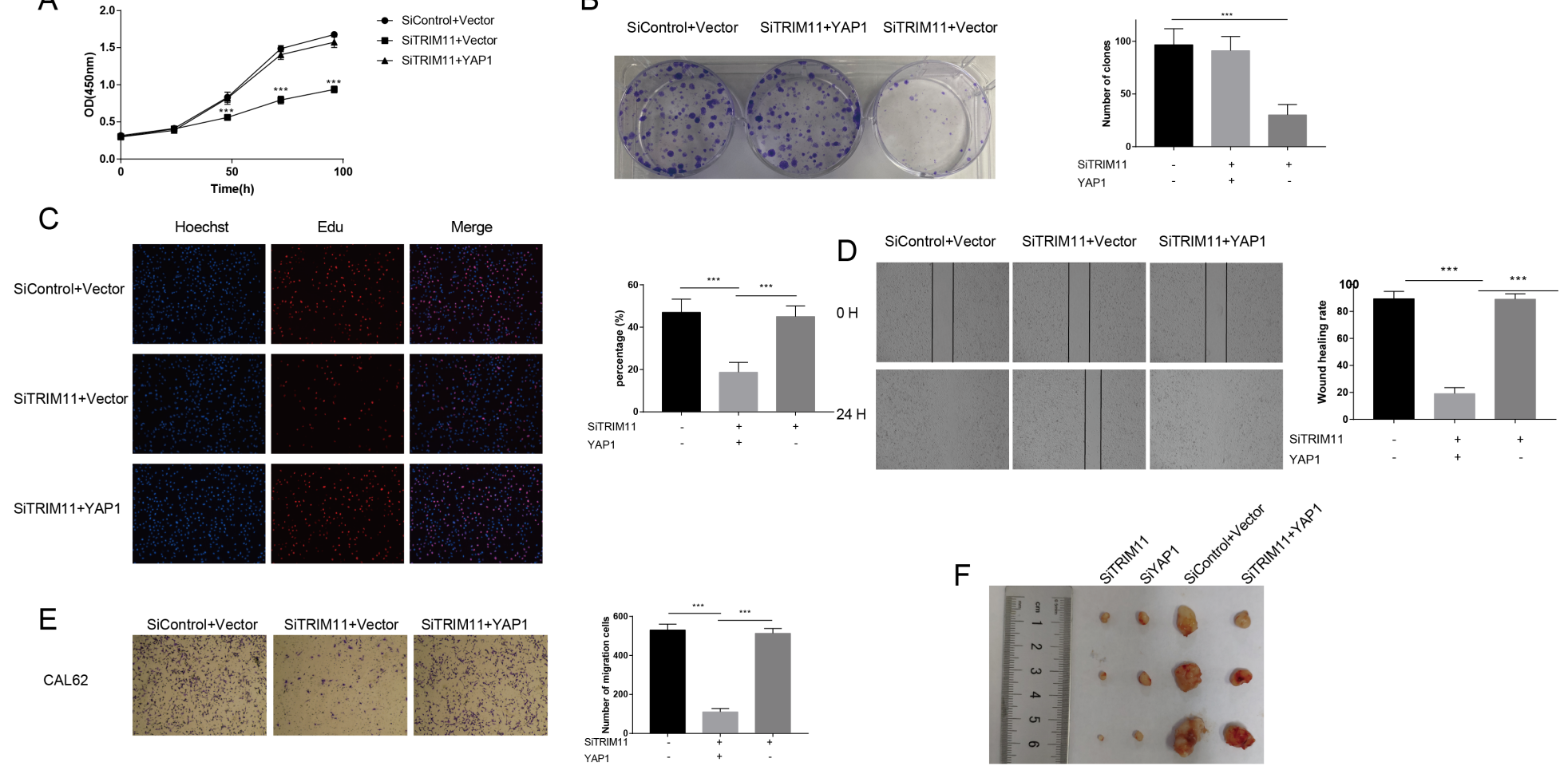

E

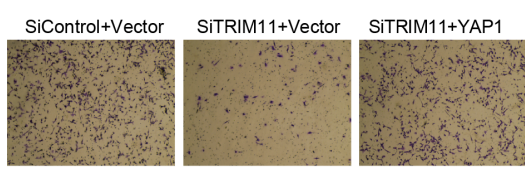

\section{Figure 6}

Increased YAP expression recovered the effect of TRIM11 depletion. (A), Cell proliferation assay of CAL62. (B). Clone formation assay of CAL62. (C). Representative images of EdU assay of CAL62. (D). Wound-healing assay of CAL62. (E). Transwell migration assay of CAL62. (F). Overexpression of YAP in TRIM11-knockdown cells partly recovered tumor growth in vivo. *, $\mathrm{P}$ value $<0.05$; $\star *, P$ value $<0.01 ; * \star \star, P$ value $<0.001$

\section{Supplementary Files}

This is a list of supplementary files associated with this preprint. Click to download.

- FigureS6.tif

- Figures6.tif

- Figures5.tif

- Figures5.tif

- Figures4.tif

- Figures4.tif

- FigureS3.tif

- FigureS3.tif

- Figures2.tif

- Figures2.tif 
- Figures1.tif

- Figures1.tif 